\title{
EMANCIPACIÓN Y ESTRATEGIAS RESIDENCIALES DE LOS JÓVENES EN MADRID.
}

\author{
Elena Martínez Goytre ${ }^{1}$ \\ Universidad Complutense de Madrid
}

Remisión Artículo: 11-9-2007

Palabras Clave: juventud, emancipación, estrategia residencial, esfuerzo.

Resumen: Los cambios experimentados en el mercado de vivienda durante los últimos años en Madrid han contribuido a reconsiderar tanto el funcionamiento del propio mercado como las consecuencias sociales, económicas y políticas que plantea.

El impacto de esos cambios sobre los grupos sociales no es homogéneo, siendo de destacar el diferente efecto que tiene sobre los grupos de edad. Mientras que los propietarios de una vivienda contemplan el incremento de valor de su patrimonio inmobiliario, los que viven en alquiler, y especialmente los que acceden por primera vez al mercado, se encuentran con que acceder a un bien básico como es la vivienda se convierte en una empresa cada vez más difícil. Se trata, por tanto, de analizar lo que supone para los jóvenes madrileños el acceso a la vivienda y los efectos sobre sus proyectos vitales.

\section{Introducción}

En las últimas décadas no sólo se ha experimentado un retraso en la edad de emancipación de los jóvenes en la Comunidad de Madrid y en España en general sino que ha habido transformaciones más profundas en este proceso.

El encarecimiento de la vivienda en la región madrileña, las transformaciones en el mercado laboral en el que deben insertarse los jóvenes, el alargamiento del tiempo que los jóvenes dedican a completar sus estudios, los cambios en las relaciones familiares, los cambios en los tipos de hogar que forman a la hora de establecerse de manera independiente, en los valores o la falta de seguridad necesaria para embarcarse en un proyecto de tal envergadura aparecen como posibles causas de estas transformaciones.

El proceso de emancipación del hogar de origen se ha diversificado, se ha individualizado, y no todos los jóvenes lo desarrollan de la misma manera. Para poder comprender mejor las causas que están detrás de estos cambios es necesario entender como afronta la emancipación un colectivo tan heterogéneo como es el de la juventud prestando especial atención a las diferencias internas que se puedan encontrar.

\section{Juventud y emancipación}

Para poder analizar los procesos de emancipación residencial de los jóvenes es necesario, en primer lugar, definir lo que entendemos por jóvenes y lo que entendemos por emancipación, como los dos principales elementos del objeto de estudio. El primero de ellos se refiere a los agentes de este fenómeno social mientras que la emancipación es el fenómeno en si mismo, o la acción llevada a cabo por dichos agentes. Este fenómeno sucede dentro de un contexto y unas estructuras que van a condicionar enormemente su desarrollo. Por tanto es necesario

\footnotetext{
${ }^{1}$ Persona de contacto Elena Martínez, correo: elenmart@cps.ucm.es
} 
contextualizar y definir estos dos elementos teniendo en cuenta las estructuras en las que se hallan inmersos actualmente.

Comenzando por el sujeto de la acción, jóvenes, como plural de joven, se refiere a las personas que están en la juventud. ${ }^{2}$ Por tanto, lo que aquí se debe tratar de definir, es lo que entendemos por juventud.

No es una tarea fácil, y ha habido múltiples y variadas definiciones de dicho concepto dentro de la denominada "sociología de la juventud". Enumerar y presentar aquí todas las definiciones que se han hecho es a la vez innecesario e imposible debido a la extensa bibliografía que se puede encontrar dentro de esta rama de la sociología. Se trata más bien de llegar a comprender a que nos referimos cuando hablamos de juventud, teniendo en cuenta las principales perspectivas y enfoques desde los que se ha construido este concepto.

El primer problema que nos encontramos a la hora de definir juventud lo plantea Martín Criado en la definición que hace sobre el concepto en el Diccionario Crítico de las Ciencias Sociales. En esta definición el autor se hace la siguiente pregunta "¿En virtud de que "formidable abuso del lenguaje" se puede pasar de una identidad de edad biológica a una identidad de conformación de "opiniones", de "actitudes", de situaciones: de sujetos?". Lo que defiende a la hora de definir juventud es que no se trata de un grupo social ni una categoría homogénea y plantea que bajo la identidad del nombre de juventud se agrupan sujetos y situaciones que solo tienen en común la edad. Esta falta de homogeneidad genera un claro problema a la hora de definir el concepto. Efectivamente, dentro de lo que comúnmente conocemos por juventud, existe una gran variedad de grupos sociales bien diferenciados, como pueden ser las diferencias marcadas por la clase social o el género. Podemos aceptar, por tanto, que al hablar de juventud no estamos hablando de grupo social, por lo que inevitablemente debemos cuestionarnos a qué nos referimos.

Sin embargo, a esa etapa del ciclo vital le corresponde una determinada función social. En esta idea es en la que se basan otros muchos autores que entienden que detrás de la palabra juventud existe algo más que una mera cuestión de calendario. Como defiende Zárraga "no puede dejar de hablarse de "juventud" para hablar concretamente de "los jóvenes", porque los jóvenes no son sólo un estrato de la población -como cualquier otro que definamos por la edad- sino sujetos de una condición social específica y los protagonistas de un proceso esencial en la reproducción de la sociedad, que es precisamente lo que llamamos "juventud". Si los consideramos sólo como "jóvenes", es decir, como individuos con una determinada edad ignoramos todo lo que de específico tiene su situación en la sociedad" (Zárraga, 1985:3). La existencia de esta función social nos remite al concepto de condición social, entendiendo como tal "el conjunto de estatutos que asume y de funciones sociales que desempeña una categoría de sujetos en una sociedad. Desde el punto de vista del individuo, la "condición social" es un sistema de determinaciones, límites y constricciones que se le imponen socialmente, en cuanto individuo perteneciente a unas categorías sociales específicas" (Zárraga, 1985:5).

Al hablar de juventud estamos hablando también de un proceso social en el que "se pasa de la dependencia propia de la infancia a la independencia más propia de la edad adulta" (López Blasco, 2005:7), de un proceso social de emancipación que atañe aspectos económicos y familiares (Casal, García, Merino y Quesada, 2006:21).

Dichos procesos de emancipación familiar vendrán determinados por las estructuras en las que los jóvenes se hayan inmersos, jóvenes que en muchos casos conforman generaciones diferenciadas de las de sus progenitores y en los que se puede observar un cambio de valores culturales y sociales. Pero no se debe perder de vista en el análisis de esos procesos la cualidad de los jóvenes como agentes sociales, que llevan a cabo su transición desde la infancia hacia una mayor autonomía a través de diferentes trayectorias vitales. Y estas trayectorias o itinerarios, concepto clave dentro de la perspectiva biográfica, "tienen un vínculo

\footnotetext{
${ }^{2}$ Definición de "joven" del Diccionario de la Real Academia de la lengua.
} 
con la estructura social y las segmentaciones, no son independientes de las clases sociales, ni del género, ni de la etnia, ni de las migraciones." (Casal, García, Merino y Quesada, 2006:34).

Por tanto, parece adecuado abordar los estudios sobre juventud desde un enfoque que considere las estructuras en las que se halla inmerso el joven, pero que no caiga en un determinismo absoluto de estos elementos sobre los procesos de transición de los jóvenes y considere además otra serie de factores que tienen que ver con las elecciones racionales de las personas, con las decisiones que vayan tomando.

Para explicar los cambios que se están produciendo en los procesos de emancipación de los jóvenes se ha recurrido durante los últimos años a diversos conceptos. Sin embargo, antes de adentrarnos en los cambios conviene aclarar brevemente lo que en el marco de este estudio se entiende por emancipación.

En primer lugar habría que distinguir dos tipos diferentes de emancipación: por un lado la "emancipación familiar completa" o "emancipación juvenil", que entraría dentro del propio concepto de juventud como la situación que marca la finalización del mismo. Se entiende que se ha alcanzado una emancipación familiar completa en el momento en que se adquiere una total autonomía con respecto a la familia de origen. Por otro lado estaríamos hablando de "emancipación residencial" refiriéndonos a "la transición que supone el abandonar por primera vez en la vida el domicilio donde se estaba conviviendo desde siempre con el padre y/o la madre para formar un hogar independiente" (Miret Gamundi, 1997).

En numerosas ocasiones la emancipación residencial coincide con la emancipación familiar completa y la entrada en la vida adulta, ya que para poder emanciparse residencialmente es necesario disponer de los recursos económicos necesarios para poder hacer frente a los gastos de la vivienda y de una vida en un hogar independiente. Para poder disponer de esos recursos lo más común es que el joven haya alcanzado previamente autonomía económica a través de un empleo. Además, para analizar la relación de los jóvenes con el mercado de la vivienda el objeto de interés es la emancipación residencial.

El análisis del proceso de emancipación a través de las fuentes secundarias disponibles es muy complicado. No podemos dibujar las trayectorias de los jóvenes ni podemos analizar el momento exacto en el que el joven se establece en un hogar independiente. Lo único que podemos saber es que cuáles son sus características según su condición de emancipados o no. Pero este tipo de análisis junto con un análisis cualitativo que nos permita acercarnos a las estrategias de los jóvenes pueden arrojar mucha luz sobre la relación entre el mercado de la vivienda y los procesos de emancipación juvenil.

\section{El proceso de emancipación en la actualidad}

Una vez hecha esta aclaración nos adentramos en las transformaciones que ha experimentado el proceso de emancipación juvenil. En la literatura más reciente sobre la materia vemos que se habla de "desestandarización" de las transiciones, del paso de trayectorias "lineales" a trayectorias "no lineales", de "individualización" y de mayor "complejidad" en las mismas, a la vez que se habla de retraso de emancipación o lo que es lo mismo, "alargamiento de la juventud".

De todas estas afirmaciones, la única que cuenta con evidencias empíricas irrefutables es el retraso de la emancipación. Los jóvenes permanecen durante más tiempo en casa de sus padres o en sus hogares de origen y posponen la decisión de establecerse de manera independiente. Pero la mayor complejidad, la menor linealidad o una mayor individualización y la desestandarización necesitarían, como bien apuntan Furlong, Cartmel y Biggart (2006), de estudios sobre los procesos de transición en décadas y generaciones anteriores desde una revisada perspectiva teórica y metodológica. 
Sin embargo, cabe pensar que estas afirmaciones están en lo cierto teniendo en cuenta, sobre todo, los cambios introducidos en los modos de producción, por una "nueva división del trabajo resultante de la reconversión tecnológica que ha destruido el tejido ocupativo en que se insertaban las familias" (Gil Calvo, 2002:7), que han provocado una mayor incertidumbre y han aumentado la percepción del riesgo. Se trata de incertidumbre, tal y como explican Casal, García, Merino y Quesada (2006b:217), en las formas de transición de la educación al trabajo en la relación entre los estudios realizados y las oportunidades de empleo reales-, a la hora de decidirse a embarcarse en una hipoteca o sistemas de financiación a largo plazo, o a la hora de confiar en la estabilidad para formar un hogar con una pareja.

Esta incertidumbre acaba afectando las decisiones de los jóvenes y, por tanto, las trayectorias que siguen en sus transiciones. Ante esta situación, estos mismos autores hacen hincapié en el concepto de "testing" o "prueba", que aparece ante la complicada decisión sobre qué estudiar, sobre la elección del primer trabajo en un panorama en el que tienen muchas opciones pero con poca proyección de futuro, en las decisiones sobre emancipación relacionadas con compromisos financieros y emocionales a largo plazo (Casal, García, Merino y Quesada, 2006b:217).

Los jóvenes no tienen una guía clara sobre como llevar a cabo sus transiciones y en algunos casos se encuentran cambiando de decisiones después de intentos y pruebas fallidas en sus caminos hacia la independencia. Esto nos lleva a pensar que es probable que haya aumentado la no linealidad de las transiciones, su desestandarización, individualización y su complejidad.

De cualquier modo, sea cual sea este supuesto incremento, Furlong, Cartmel y Biggart (2006) plantean dos maneras de interpretar la complejidad en las transiciones juveniles. Critican la tesis de Du Bois Reymond sobre las "choice biographies", asociados con los enfoques de reflexividad y live management. Ellos consideran que la mayor complejidad, por el contrario, puede estar asociada a una falta de opciones y a los efectos de la flexibilización del mercado de trabajo. Y plantean que se deben distinguir los cambios provocados por la precariedad laboral de aquellos que sugieren flexibilidad. Consideran, por tanto, que la complejidad está asociada a nuevas formas de vulnerabilidad y a la percepción del riesgo y de la incertidumbre (Furlong, Cartmel y Biggart, 2006:227).

Efectivamente, la inestabilidad y la precariedad laboral traen consigo transiciones más complejas, en las que los jóvenes cambian de estatus en numerosas ocasiones, pudiendo dar lugar a lo que se han denominado trayectorias yo-yo (Du Bois Reymond y López Blasco, 2004). ${ }^{3}$

Pero no siempre se trata de una falta de opciones. En algunos casos los jóvenes vuelven a los estudios como parte de una estrategia de mejora laboral. La necesidad de mejorar su formación después de haberse adentrado en el mercado laboral en algunos casos está provocada por la incertidumbre sobre la adecuación de su formación a los perfiles laborales exigidos, pero puede ser que responda también a que el joven se encuentra insatisfecho con su vida laboral y decide reorientarla de nuevo a través de la vuelta a los estudios. En este caso tendría que ver con los efectos de la incertidumbre y el concepto de testing, pero no con una falta de opciones o de posibilidades.

Sin embargo, es importante hacer hincapié en el hecho de que no todos los jóvenes disponen de las mismas oportunidades a la hora de definir sus estrategias, que son una "selección de

\footnotetext{
${ }^{3}$ Este concepto ha sido criticado desde el GRET alegando que no se cumple el requisito de reversibilidad que establece. Desde este grupo se plantea más bien que existe una cristalización o un bajo nivel de reversibilidad en las decisiones que van tomando los jóvenes a lo largo de su transición. Sin embargo, entiendo que se puede hablar de reversibilidad en tanto que se puede pasar a la fase previa, desde el punto de vista de una transición lineal (formación-trabajo-vivienda), aunque al volver a la fase previa no se estén necesariamente eliminando los efectos que los pasos anteriores tuvieron sobre la trayectoria vital del joven.
} 
cursos alternativos (recursos tácticos) por su virtualidad para producir resultados futuros (objetivos estratégicos) en situaciones de incertidumbre" (Garrido Medina y Gil Calvo, 1993:14). Estas estrategias vendrán determinadas tanto por los recursos de los que dispongan como por las preferencias que guiarán los objetivos que se marquen.

Se parte, pues, de la idea de que los procesos de emancipación están condicionados por las estructuras pero "este dominio nunca es absoluto y siempre hay un espacio para la acción viable y la subjetividad viable en la coyuntura." (Heller, 1977) ${ }^{4}$. Se puede afirmar, de manera esquemática, que las estructuras condicionan la acción individual, pero que dejan todavía un margen de maniobra dentro del cual los agentes sociales cobran protagonismo como sujetos activos con cierto poder de decisión sobre sus comportamientos o prácticas, que a su vez tienen el poder de transformar las estructuras pre-existentes. Pero, partiendo de esta premisa, el interés del estudio se centra sobre todo en las estructuras.

Dentro de estas, los dos elementos que afectan de manera más directa a los procesos de emancipación, ya que son los instrumentos fundamentales de adquisición de la autonomía, son el mercado laboral y el mercado residencial. A través del mercado laboral adquieren una autonomía económica y a través del mercado de vivienda una autonomía residencial. Pero también existen otras estructuras que afectan estos procesos, como pueden ser la familia, las redes informales, las instituciones públicas o la cultura.

En este caso el análisis se centra en el modo en que los jóvenes se insertan en el mercado residencial y las estrategias que desarrollan, atendiendo tanto a sus preferencias como a los recursos de que disponen. No disponemos de datos desagregados sobre los recursos económicos de los jóvenes pero si podemos hacer un análisis a partir de su condición socioeconómica estableciendo tres categorías: directivos y profesionales, nivel intermedio y trabajadores manuales.

\section{Mercado de trabajo y emancipación}

Según aparece en el Informe de Juventud en España 2004, los dos requisitos que los jóvenes consideran más importantes para poder emanciparse son el empleo (65\%) y la vivienda (15\%).

Al analizar la evolución de la ocupación se constata que, tanto en España como en Madrid, ha habido un aumento de los ocupados en el grupo de los jóvenes. La evolución en ambos casos es similar: hasta mitad de la década de los noventa ha habido periodos en que la tasa de ocupación ha descendido entre todos los jóvenes independientemente de que hayan alcanzado la independencia residencial o no, y a partir de 1995 la recuperación de la ocupación es continua. Por otro lado, hay que señalar, que al mayor porcentaje de ocupados en la Comunidad de Madrid le corresponde una emancipación más tardía que en España.

Tabla 1 Tasa de ocupados entre los emancipados y los no emancipados jóvenes de España y la Comunidad de Madrid entre 1980 y 2004.

\footnotetext{
${ }^{4}$ Citado por Enrique De la Garza Toledo en "Subjetividad, Cultura y Estructura", Revista Iztapalapa, n 50, 2001 México, pp. 83-104.
} 


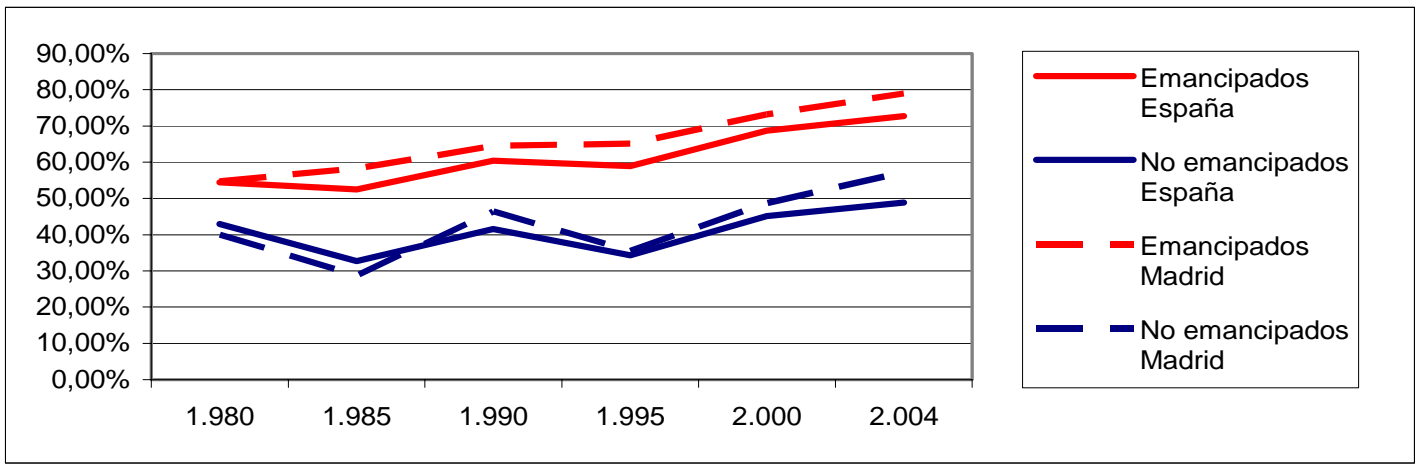

Fuente: elaboración propia a partir de la EPA.

Además, aumenta en mayor medida la tasa de ocupación en el grupo de no emancipados en Madrid. El hecho de que los jóvenes dispongan de un trabajo no implica que decidan emanciparse ni que puedan hacerlo, ya que más de la mitad de los no emancipados en la Comunidad de Madrid están ocupados.

Parece que "cuando el paro de los jóvenes crece, la emancipación se retrasa. Pero esta ecuación no es reversible, pues puede no cumplirse a la inversa. Así sucede ahora, cuando la reactivación de la tasa de ocupación juvenil, ocurrida desde 1995 no ha sido capaz de invertir la tendencia, favoreciendo el adelanto de la edad emancipatoria. ¿Por qué? Sin duda porque el empleo que se ha creado es de muy mala calidad, dada su extrema temporalidad." (Gil Calvo, 2002:1).

El porcentaje de contratos temporales entre los jóvenes ha aumentado. El único grupo en que han disminuido es en los no emancipados del conjunto del territorio español, pero siguen siendo el grupo con mayores tasas de temporalidad.

Gráfico 1 Porcentaje de contratos temporales entre los jóvenes ocupados emancipados y no emancipados en España y en la Comunidad de Madrid, 1990 y 2004.

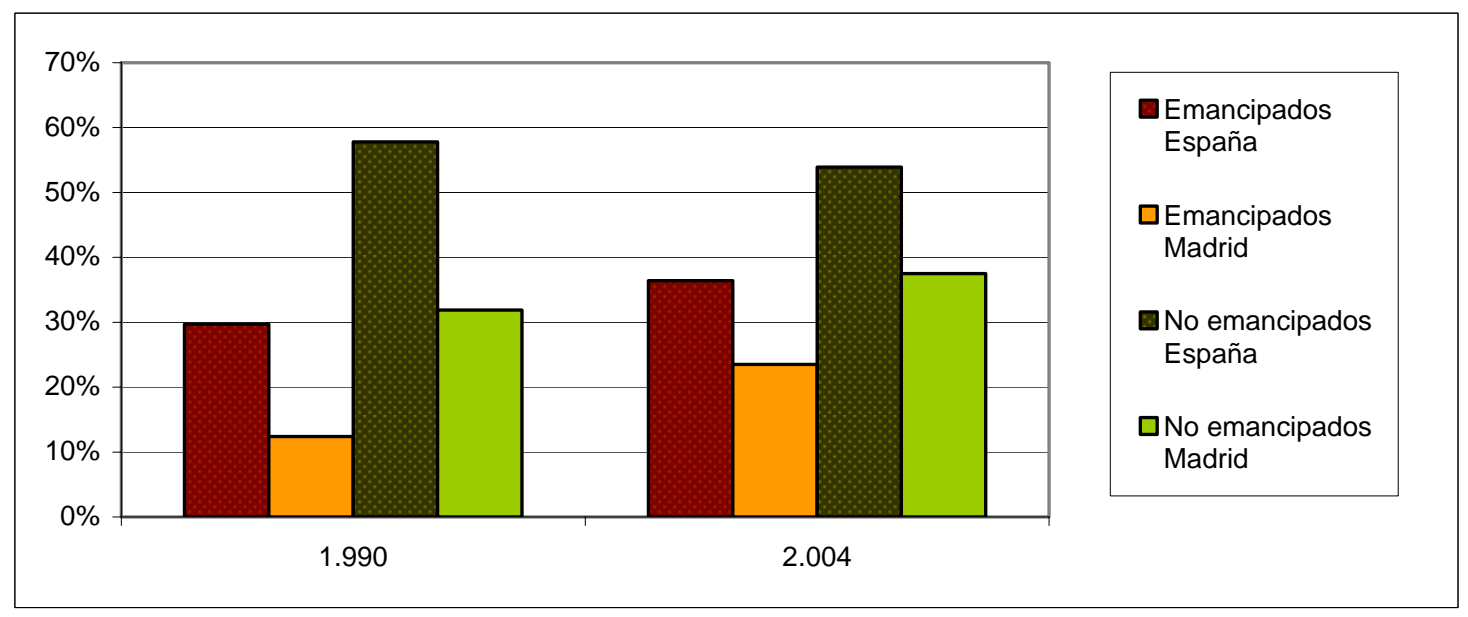

Fuente: elaboración propia a partir de la EPA. 
Por otro lado, es evidente que Madrid no es una de las Comunidades con mayores niveles de inestabilidad ya que se sitúa bastante por debajo de la media española. Dentro del contexto español, Madrid tiene un mercado laboral bastante más estable que la media, aunque la inestabilidad ha aumentado bastante en los últimos quince años, especialmente en el grupo de los emancipados. De cualquier modo, este grupo tiene un porcentaje de contratos temporales inferior al de los que permanecen en el hogar de origen con lo que la relación entre la estabilidad laboral y el proceso de emancipación queda patente.

La estabilidad laboral afecta el proceso de emancipación como refleja la mayor tasa de temporalidad de los jóvenes que viven todavía con sus padres. Pero el aumento de la inestabilidad en un contexto de descenso de las tasas de desempleo puede estar provocando que los jóvenes sientan una mayor confianza en que después de ese contrato volverán a tener otro sin demasiada dificultad. Puede que sea este hecho el que esté detrás de la reducción de las diferencias en la temporalidad entre los emancipados y los no emancipados.

De cualquier modo la inestabilidad sigue afectando a los jóvenes en sus procesos de emancipación. Aunque la estabilidad no sea un garante de la emancipación, desde luego su ausencia si es una dificultad para la misma.

\section{El problema residencial}

El mercado residencial afecta directamente a los procesos de emancipación juvenil ya que acceder a una vivienda es un paso ineludible. Por tanto, es necesario analizar como ha evolucionado el esfuerzo de acceso a la vivienda que realizan los jóvenes, entendiendo por esfuerzo el porcentaje de los ingresos -en el caso de los jóvenes generalmente procedente de los salarios en su mayor parte- destinados a costearse una vivienda.

Pero antes de adentrarnos es esta cuestión es necesario señalar que dentro del mercado residencial existen dos mercados bien diferenciados: el de compra-venta y el de alquiler. La Comunidad de Madrid se caracteriza por ser una región de propietarios. La mayor parte de los jóvenes accede a sus viviendas también a través de la propiedad pero en este caso la tasa de alquiler es muy superior a la media.

Tabla 2 Régimen de tenencia de las viviendas en la Comunidad de Madrid según la edad del cabeza de familia, 2001

\begin{tabular}{|l|c|c|c|c|c|}
\hline Intervalos de edad & $\begin{array}{l}\text { Total } \\
\text { propiedad }\end{array}$ & Alquiler & $\begin{array}{c}\text { Cedida } \\
\text { gratis }\end{array}$ & Otra & Total \\
\hline 16a19 & 55,2 & 36,7 & 1,9 & 6,2 & 100 \\
\hline 20 244 & 52,1 & 39,3 & 3,6 & 5,1 & 100 \\
\hline $25 a 29$ & 66,5 & 26,7 & 3,1 & 3,8 & 100 \\
\hline 30a34 & 74,9 & 19,0 & 2,7 & 3,4 & 100 \\
\hline Total jóvenes 16 a 34 & 69,8 & 23,6 & 2,9 & 3,7 & 100 \\
\hline Total población & 82,0 & 13,6 & 1,9 & 2,5 & 100 \\
\hline
\end{tabular}

Fuente: Censo 2001

Sin embargo, a la hora de analizar el esfuerzo de acceso al alquiler nos encontramos con un problema de falta de información. No disponemos de bases de datos sistematizadas sobre precios de alquiler y la única información disponible es la de su incremento ${ }^{5}$ que tampoco

\footnotetext{
${ }^{5}$ Los datos disponibles sobre los precios de alquiler son los datos que obtenemos a partir del IPC obtenido a partir de la Encuesta de Presupuestos Familiares, en el que aparece el incremento del precio de la vivienda en alquiler. Sin embargo estos datos no resultan fiables debido a que el alquiler mensual que pagan los hogares se ve alterado al incluir en esta categoría una estimación del propio encuestado que ha accedido a su vivienda a través de la compra
} 
resulta fiable. Aunque estamos en un contexto en el que la propiedad es el régimen predominante, el alquiler tiene especial importancia a la hora de hablar de los problemas residenciales de los jóvenes. Sin poder realizar un análisis sobre la evolución del esfuerzo de acceso al alquiler sólo nos queda trabajar con la hipótesis de que el precio del alquiler ha aumentado enormemente en los últimos años en la Comunidad de Madrid debido a la escasa oferta de viviendas bajo este régimen, empujadas a su vez por el alza de la vivienda en venta, hipótesis que parecen confirmar los testimonios de los jóvenes que han participado en el trabajo cualitativo desarrollado en el marco de esta investigación. A partir de aquí, el análisis de la evolución de los precios y del esfuerzo de acceso a la vivienda se va a centrar sobre todo en el mercado de compra-venta.

Gráfico 2 Evolución del precio del metro cuadrado de las viviendas en España y en Madrid.

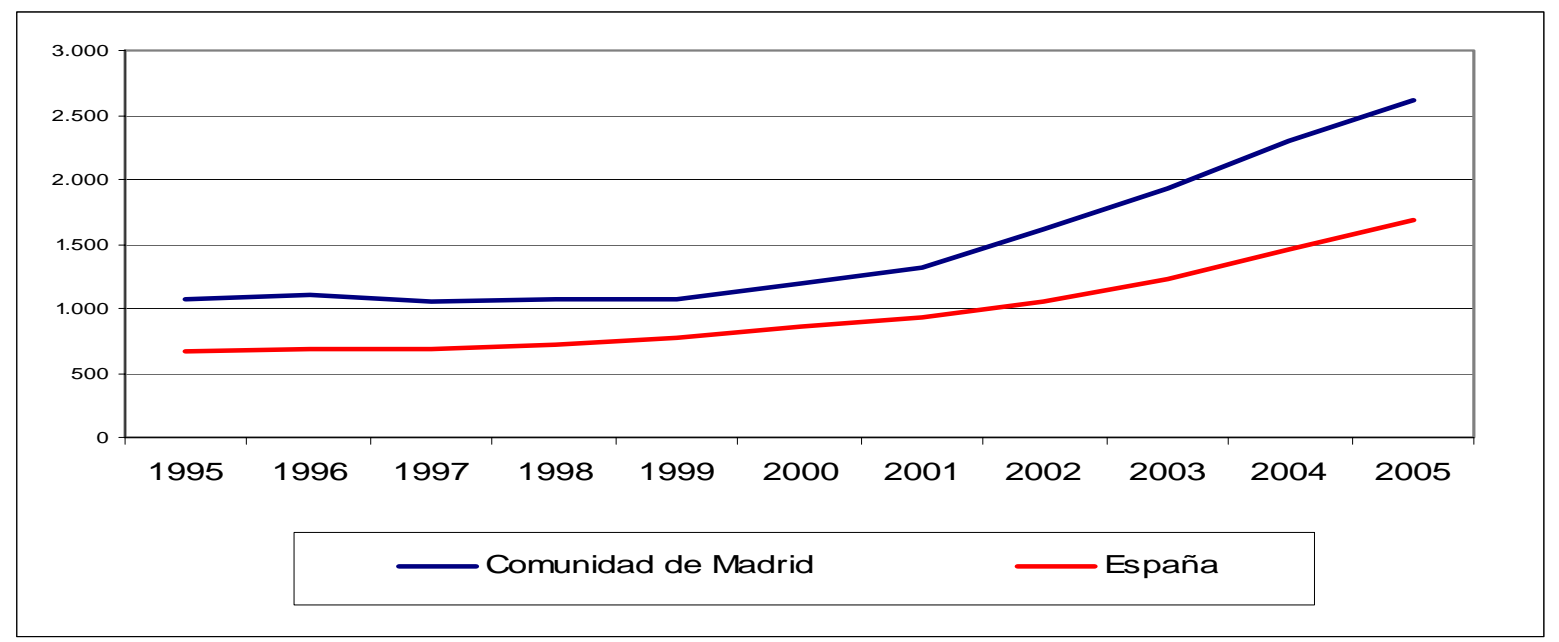

Fuente: Ministerio de Vivienda.

El primer elemento a tener en cuenta a la hora de analizar el esfuerzo es la evolución en el precio de la vivienda. Éste ha aumentado de forma continuada desde 1995 y de forma más acusada a partir de 1999. Hay que señalar que la subida de precios de las nuevas viviendas que se construyen han arrastrado al alza también los precios de las viviendas de segunda mano, que han superado las tasas de incremento de las primeras.

\section{Gráfico 3 Incremento relativo del precio por $\mathbf{m} 2$ de la vivienda en la Comunidad de Madrid}

sobre lo que considera que pagaría por esa vivienda al mes en caso de estar en régimen de alquiler. Esta estimación distorsiona enormemente los datos si tenemos en cuenta que la proporción de hogares en propiedad es del 82\% y resulta demasiado aventurado afirmar que las estimaciones de los propietarios se adecuan a los cambios reales que sufre el mercado de alquiler. 


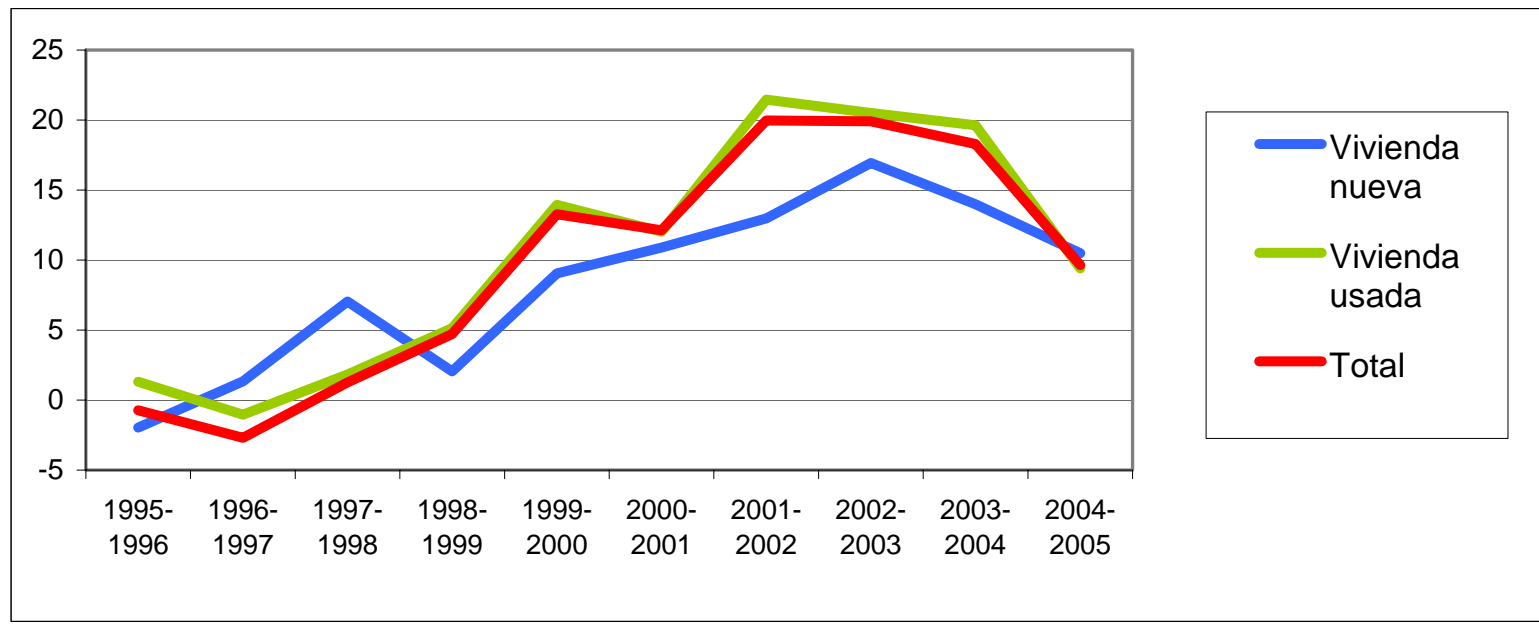

Fuente: Ministerio de la Vivienda.

Los elevados precios de la Comunidad son los causantes de ese mayor esfuerzo de acceso a la vivienda. No podemos atribuir esta diferencia al mercado de trabajo ya que los madrileños reciben mejores salarios que la media.

Tabla 3 Esfuerzo de los jóvenes en la Comunidad de Madrid, 2002

\begin{tabular}{|l|c|c|c|c|c|c|c|}
\hline Jóvenes & $\begin{array}{l}\text { Salarios } \\
\text { medios } \\
\text { mensuales }\end{array}$ & $\begin{array}{l}\text { precio m2 } \\
\text { vivienda }\end{array}$ & $\begin{array}{l}\text { Precio } \\
\text { medio de la } \\
\text { vivienda }\end{array}$ & Intereses & $\begin{array}{l}\text { Plazo de } \\
\text { amortización }\end{array}$ & $\begin{array}{l}\text { cuota del } \\
\text { préstamo }\end{array}$ & esfuerzo \\
\hline $\begin{array}{l}\text { Menos de 20 } \\
\text { años }\end{array}$ & 749,6 & $1.612,3$ & 103832,12 & 4,74 & 21 & 651 & 86,8 \\
\hline $\begin{array}{l}\text { De 20 a 29 } \\
\text { años }\end{array}$ & 1298,7 & $1.612,3$ & 103832,12 & 4,74 & 21 & 651 & 50,1 \\
\hline $\begin{array}{l}\text { De 30 a 39 } \\
\text { años }\end{array}$ & 1978,6 & $1.612,3$ & 103832,12 & 4,74 & 21 & 651 & 32,9 \\
\hline
\end{tabular}

Fuente: Elaboración propia a partir de Encuesta de Estructuras Salariales, Encuesta de Población Activa y Banco de España.

\footnotetext{
${ }^{6} 80 \%$ del valor total medio de una vivienda de 80,5 m2 (media de la vivienda joven en España en la Comunidad de Madrid, 2001).
} 
Tabla 4 Esfuerzo de los jóvenes en España, 2002

\begin{tabular}{|l|c|c|c|c|c|c|c|}
\hline Jóvenes & $\begin{array}{c}\text { Salarios } \\
\text { medios } \\
\text { mensuales }\end{array}$ & $\begin{array}{c}\text { Precio m2 } \\
\text { vivienda }\end{array}$ & $\begin{array}{c}\text { Precio medio } \\
\text { de la } \\
\text { vivienda }\end{array}$ & Intereses & $\begin{array}{c}\text { Plazo de } \\
\text { amortización }\end{array}$ & $\begin{array}{c}\text { cuota del } \\
\text { préstamo }\end{array}$ & esfuerzo \\
\hline $\begin{array}{l}\text { Menos de } \\
\text { 20 años }\end{array}$ & 807,2 & $1.051,7$ & 73366,592 & 4,74 & 21 & 460 & 57,0 \\
\hline $\begin{array}{l}\text { De 20 a 29 } \\
\text { años }\end{array}$ & 1180,2 & $1.051,7$ & 73366,592 & 4,74 & 21 & 460 & 39,0 \\
\hline $\begin{array}{l}\text { De 30 a 39 } \\
\text { años }\end{array}$ & 1642,3 & $1.051,7$ & 73366,592 & 4,74 & 21 & 460 & 28,0 \\
\hline
\end{tabular}

Fuente: Elaboración propia a partir de Encuesta de Estructuras Salariales, Encuesta de Población Activa y Banco de España.

En definitiva, en la Comunidad de Madrid se realiza un esfuerzo mayor que la media nacional. Pero más allá de esta diferencia, lo que nos interesa analizar aquí es la evolución del esfuerzo y su relación con la emancipación. Desde 1995 hasta 1999 ha habido una reducción del esfuerzo $^{8}$ y a partir de entonces este ha venido aumentando debido al mayor ritmo de crecimiento de los precios de vivienda.

\footnotetext{
${ }^{7}$ En este caso la superficie media es de $87,2 \mathrm{~m} 2$.

${ }^{8}$ Para estudiar la evolución del esfuerzo se ha escogido el grupo de edad de 20 a 29 años debido a que no es posible desagregar esta edad en grupos quinquenales a partir de las fuentes disponibles. El grupo anterior no se ha escogido por motivos obvios (es un grupo e el que la emancipación apenas está presente) y el siguiente porque buena parte de sus representantes han superado ya hace tiempo la emancipación y no pueden considerarse jóvenes.
} 
Tabla 5 Proyección provisional del esfuerzo de acceso a la vivienda para jóvenes de entre 20 y 29 años en la Comunidad de Madrid ${ }^{9}$

\begin{tabular}{|c|c|c|c|c|c|c|}
\hline Año & Salario & Precio medio & Intereses & $\begin{array}{c}\text { Plazo de } \\
\text { amortización }\end{array}$ & $\begin{array}{c}\text { Cuota } \\
\text { mensual }\end{array}$ & Esfuerzo \\
\hline 1995 & 984,1 & $69.448,96$ & 11,01 & 15 & 790 & 80,3 \\
\hline 1996 & 1039,4 & $71.129,80$ & 8,17 & 17 & 646 & 62,2 \\
\hline 1997 & 1107,6 & $67.929,12$ & 6,26 & 19 & 510 & 46,0 \\
\hline 1998 & 1146,0 & $68.940,20$ & 5,56 & 20 & 477 & 41,6 \\
\hline 1999 & 1173,3 & $69.281,52$ & 4,7 & 20 & 446 & 38,0 \\
\hline 2000 & 1212,2 & $77.370,16$ & 5,88 & 20 & 549 & 45,3 \\
\hline 2001 & 1244,5 & $85.123,92$ & 5,61 & 20 & 591 & 47,5 \\
\hline 2002 & 1298,7 & $103.832,12$ & 4,74 & 21 & 651 & 50,1 \\
\hline 2003 & 1381,4 & $124.736,36$ & 3,64 & 22 & 687 & 49,7 \\
\hline 2004 & 1449,5 & $147.997,64$ & 3,35 & 23 & 770 & 53,1 \\
\hline 2005 & 1515,3 & $168.560,56$ & 3,30 & 24 & 848 & 56,0 \\
\hline
\end{tabular}

Fuente: Fuente: Encuesta de Estructuras Salariales, Encuesta de Presupuestos Laborales, Encuesta de Población Activa, Banco de España y Departamento de Medio Ambiente y Vivienda.

Entre 1999 y 2004 los precios en la Comunidad de Madrid aumentan en un 143\% y en cambio los salarios sólo lo hacen en un 29\%. El hecho de que el esfuerzo haya aumentado sólo en 18 puntos con una diferencia tan grande se debe a la evolución del sistema financiero. El marcado descenso de los tipos de interés y el aumento de los plazos de amortización permiten que las cuotas mensuales que han de pagar los hogares hayan aumentado en este mismo periodo bastante menos que los precios (90\%).

Por otro lado, al considerar sólo los pagos mensuales de las hipotecas a la hora de analizar la evolución del esfuerzo se está pasando por alto el mayor esfuerzo que deben realizar los jóvenes o aquellos hogares que acceden por primera vez a la compra de una vivienda para reunir la cantidad necesaria para pagar la entrada, que suele estar en torno al $20 \%$ del precio de la misma, un precio que ha aumentado enormemente lo que supone el mismo incremento de esta cantidad. A pesar de que en algunos casos se conceden hipotecas por el valor del $100 \%$ del precio de la vivienda no es todavía un modelo de financiación generalizado y habría que tener en cuenta además que el esfuerzo mensual aumentaría sobre el elevado porcentaje que supone financiando el $80 \%$ del precio de la vivienda. Otro agravante a tener en cuenta es el aumento del tiempo que estos hogares deben hacerse cargo de sus hipotecas.

\footnotetext{
${ }^{9}$ El incremento de los salarios jóvenes se ha calculado a partir de los incrementos de los costes salariales del total de la población:

-Entre 1995 y hasta 2002 se aplicó el peso de cada incremento anual del total de la población al incremento total de los salarios jóvenes en ese periodo.

-A partir del 2002 y hasta el 2005 se ponderó el incremento de los salarios del total de la población. Para ello se calculó la diferencia en el incremento de los salarios de los jóvenes en cada grupo de edad con el incremento salarial del total de los individuos y calculando a su vez la disminución anual media en la diferencia entre ambas magnitudes partiendo de la hipótesis de que la diferencia sigue disminuyendo al mismo ritmo. por tanto la ponderación es : incremento anual salario joven es igual a el incremento anual salario total personas mas la diferencia media anual entre ambos incrementos en el periodo anterior menos la disminución anual media de la diferencia de las dos magnitudes.
} 
Gráfico 4 Proyección provisional del esfuerzo de acceso a la vivienda para personas entre 20 y 29 años y entre 30 y 39 años en la Comunidad de Madrid.

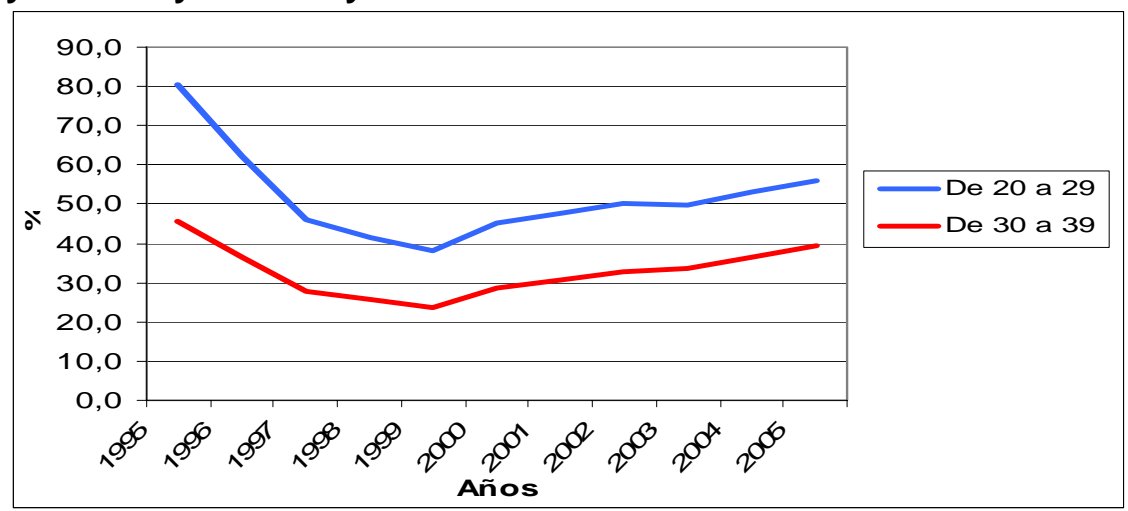

Fuente: Elaboración propia a partir de las tablas de proyecciones provisionales de esfuerzos.

Aunque no basta el mercado de vivienda para explicar el retraso en la emancipación, sí podemos afirmar que el fuerte encarecimiento de la vivienda ha provocado un aumento del esfuerzo de acceso y a la vez está reforzando las desigualdades entre los distintos grupos, llegando en algunos casos incluso al bloqueo. Además, la configuración del mercado está condicionando las estrategias residenciales de los jóvenes en estos procesos, especialmente las estrategias de acceso y de localización.

\section{Estrategias residenciales de los jóvenes}

Ya se ha comentado que los jóvenes optan en su mayoría por la compra, aunque las tasas de alquiler a estas edades son más elevadas que en las siguientes etapas del ciclo del hogar. La prevalencia de la compra se ha ido acusando a lo largo de la década de los noventa y esto se ha dejado notar en todos los grupos de edad salvo en el de menores de veinte años.

La compra parece la opción más inteligente en un contexto en el que el precio del alquiler es muy elevado y el mercado inmobiliario resulta una inversión segura y muy rentable.

Gráfico 5 Porcentaje de hogares jóvenes en alquiler según la edad de la persona de referencia, 1991 y 2001.

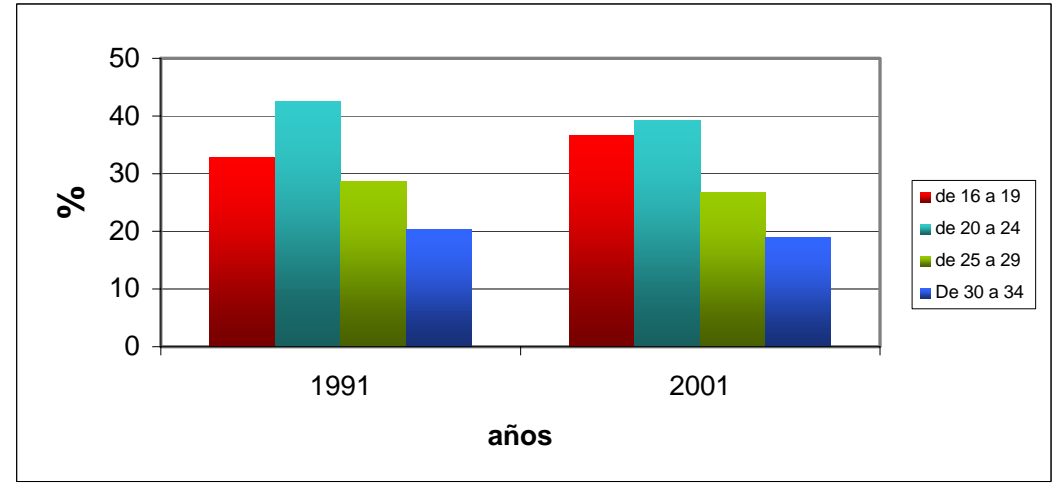

Fuente: Censo de Población y Vivienda. 
De cualquier modo, los jóvenes recurren al mercado de alquiler en mayor medida que los adultos. En muchos casos no les queda otra opción, bien sea por la falta de recursos económicos o por la inestabilidad de sus ingresos. Pero en otras ocasiones el alquiler se presenta como una elección ligada a las facilidades que este régimen ofrece en un momento de sus vidas en que todavía no tienen bien definido su proyecto profesional y familiar. La mayor flexibilidad y movilidad que ofrece el alquiler lo convierte en la opción más adecuada para algunos jóvenes, a pesar de las desventajas en términos de rentabilidad económica que conlleva. Prueba de ello es la mayor tasa de alquiler de los grupos mejor posicionados en la escala socioeconómica. ${ }^{10}$

\section{Gráfico 6 Porcentaje de hogares jóvenes en alquiler según la condición socioeconómica de la persona de referencia, 2001.}

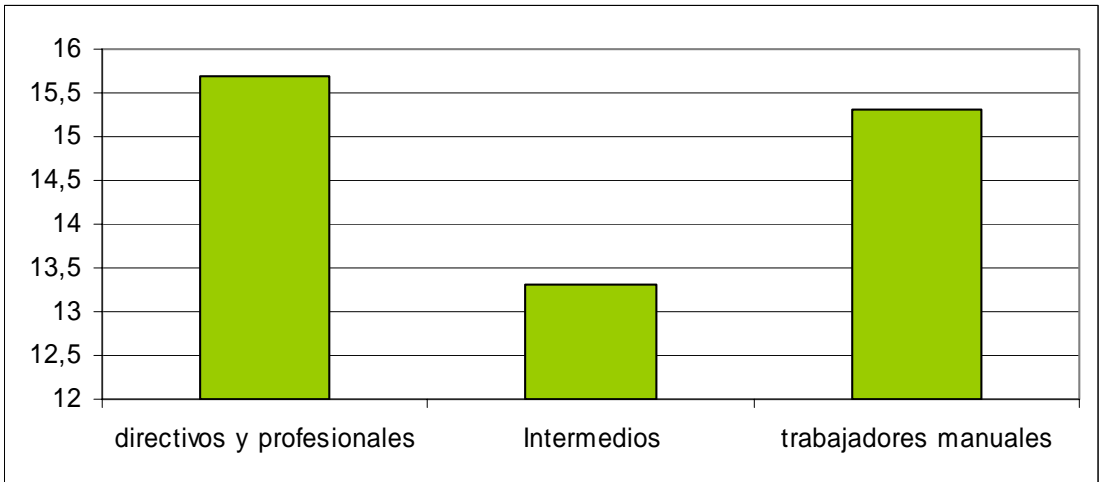

Fuente: Censo de Población y Vivienda.

Los hogares jóvenes en alquiler tienen una mayor presencia en el municipio de Madrid que en el área metropolitana y, dentro de esta, la presencia es mayor en el noroeste que en el sureste, que es la zona de mayor valor social y donde se establecen los grupos socioeconómicos más altos. El alquiler no parece ir asociado exclusivamente a los grupos con pocos recursos, sino a los dos polos de la escala socioeconómica y por motivos bien distintos como hemos visto anteriormente.

\footnotetext{
${ }^{10}$ Esta se ha clasificado en tres grupos. En el primero de ellos se han incluido todas las categorías que requieren una mayor formación y profesionalización y aquellos que han alcanzado posiciones de elevado rango, como directivos, o empresarios que cuentan además con empleados. La segunda de ellas reúne a los miembros de cooperativas, contramaestres y capataces, empresarios sin asalariados y personal administrativo y comercial. La última de ellas abarca a los trabajadores de tipo manual (operarios con o sin especialización) y al personal de servicios que no se ha incluido en la primera categoría.
} 
Gráfico 7 Porcentaje de hogares jóvenes en alquiler según el lugar de residencia, 2001.

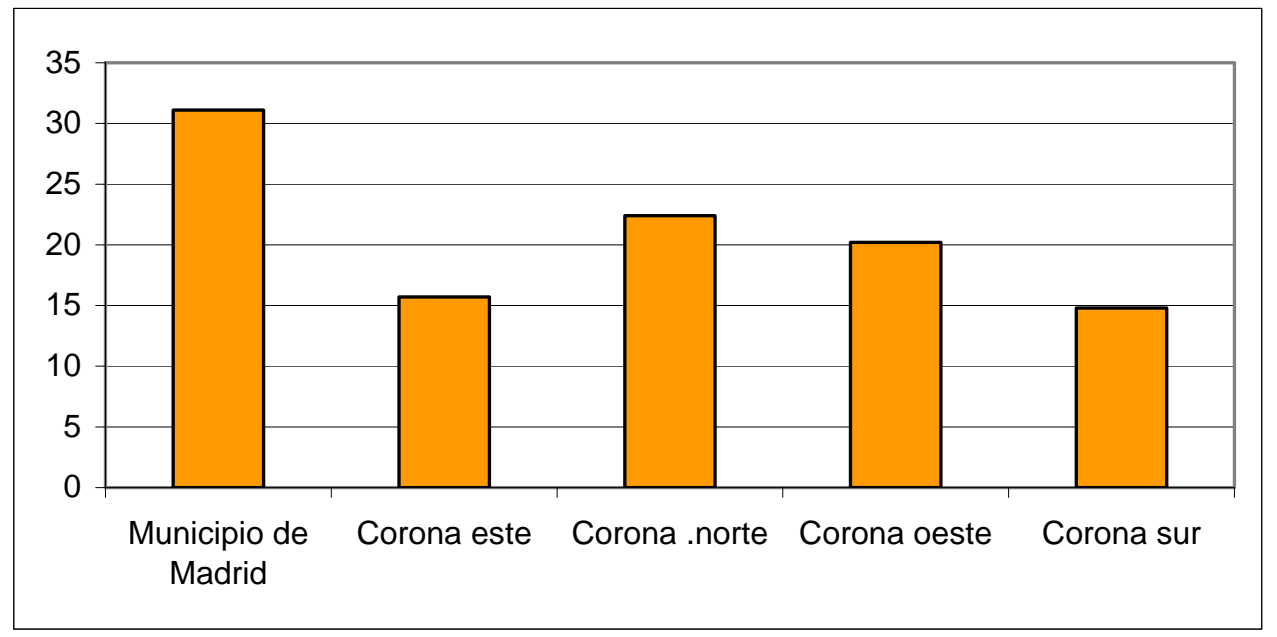

Fuente: Censo de Población y Vivienda.

Pero es en el municipio de Madrid donde encontramos las tasas más elevadas. Parece lógico si pensamos que en esta zona hay una elevada presencia de jóvenes de menor edad, que forman hogares unipersonales, multipersonales o en pareja pero sin hijos y que presenta una composición social muy heterogénea.

Gráfico 8 Localización de los hogares jóvenes atendiendo a la edad, la condición socioeconómica y el tipo de hogar, 2001.

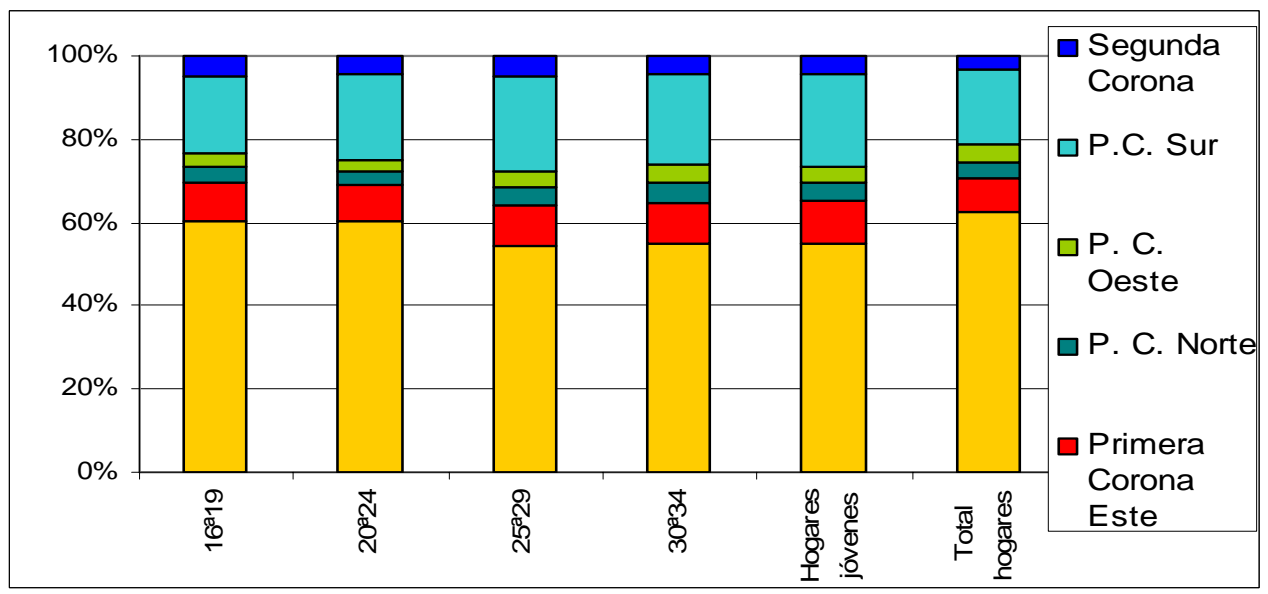



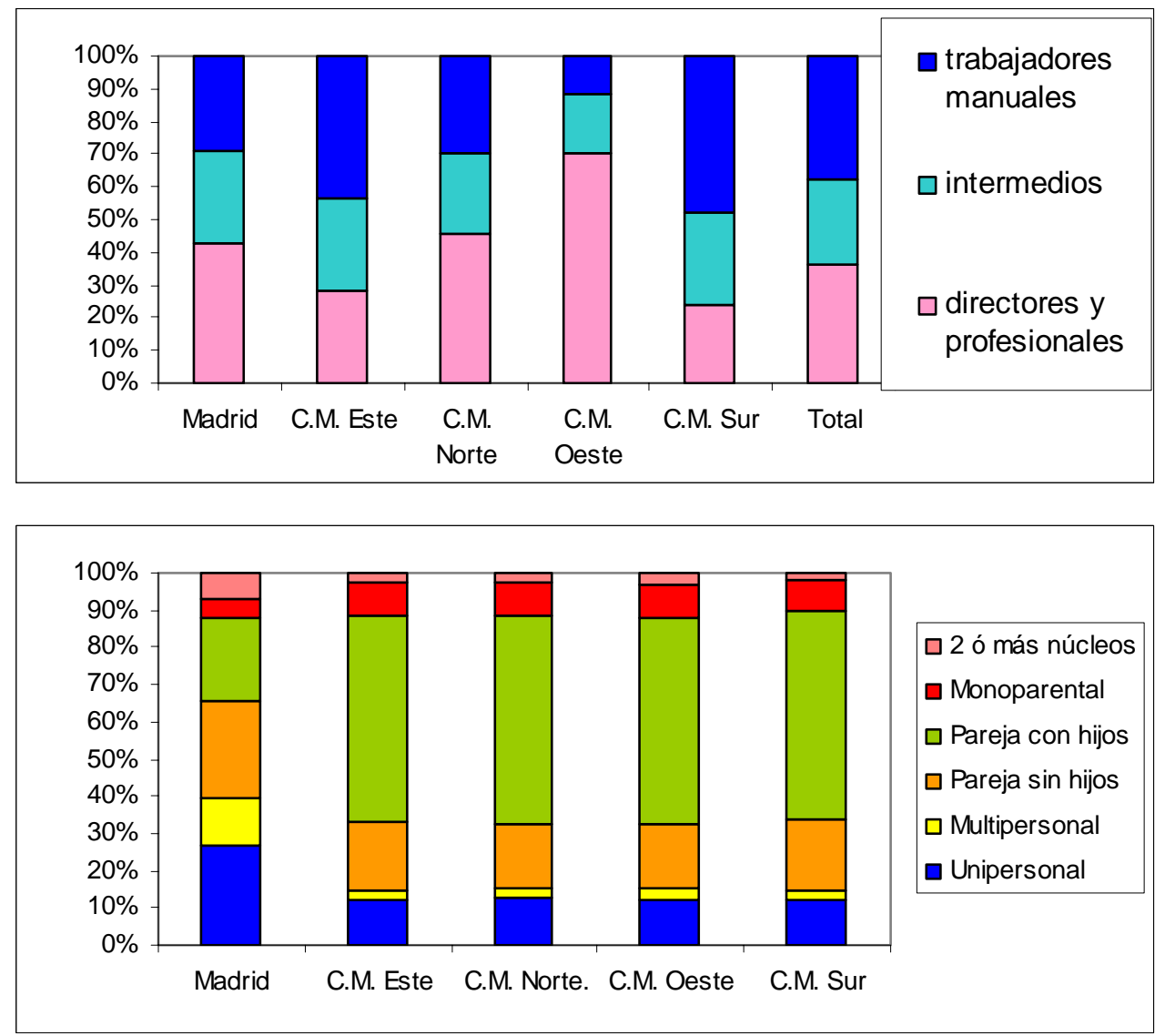

Fuente: Censo de Población y Viviendas, 2001.

La estructura de precios de vivienda de la región responde a dos pautas: la primera de ella es el valor de la centralidad; la segunda, el mayor valor social de la zona metropolitana noroeste. En definitiva, el centro y la zona noroeste de la región son las zonas con los precios más elevados.

Sin embargo, hay que señalar que el municipio de Madrid presenta un mercado residencial muy heterogéneo. Por un lado tiene un mercado de alquiler más elevado que en otras zonas de la región, y además conviven viviendas de elevado precio con un submercado más deficitario, de más antigüedad, con carencias en algunas de sus instalaciones o en mal estado, y son éstas últimas las que resultan más accesibles para los colectivos con menos recursos. 
Gráfico 9 Precio de las viviendas nuevas y usadas en cada una de las zonas de la Comunidad de Madrid en el año 2000.

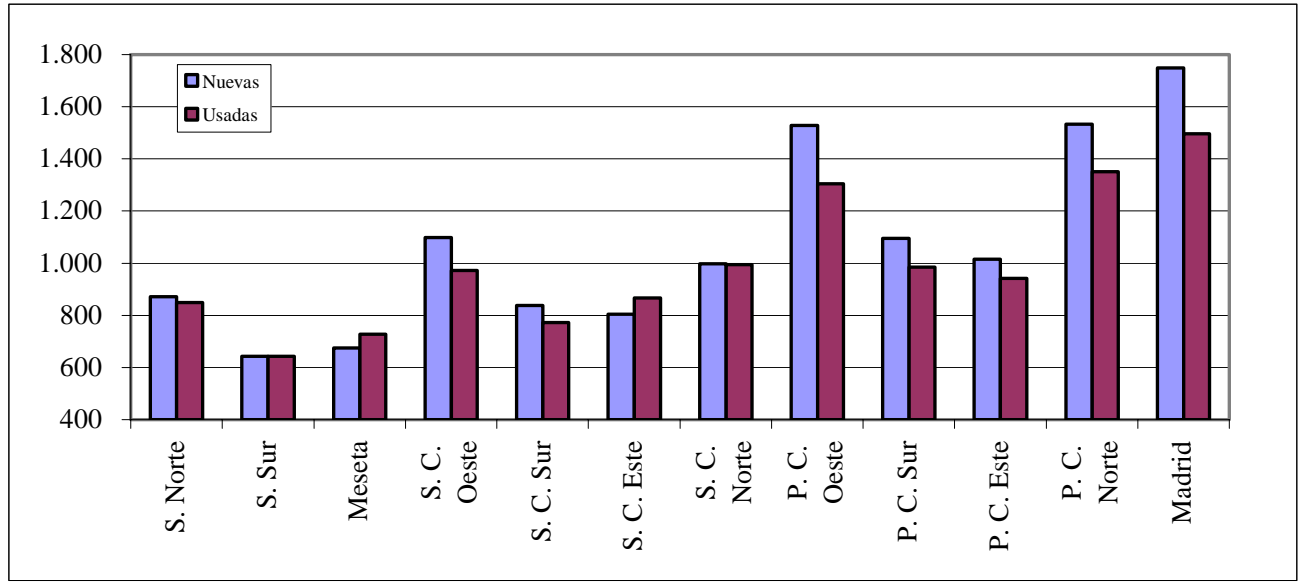

Fuente: Elaboración propia a partir de los datos del $\mathrm{M}^{0}$ de Fomento.

Esta estructura esta condicionando las estrategias de localización de los jóvenes. Si se pregunta a los jóvenes sobre cuáles son los motivos por los que desean vivir en una zona de la Comunidad los resultados son bastante claros: los jóvenes priman la cercanía a familiares y amigos y las buenas comunicaciones. Pero además, hay algunas diferencias entre los distintos grupos de jóvenes que conviene destacar. Los de mayor nivel socioeconómico son más independientes de sus redes sociales y valoran más la centralidad que los grupos de menor nivel.

Tabla 6 Motivos de interés en las zonas de búsqueda de los demandantes jóvenes en la Comunidad de Madrid, 2003.

\begin{tabular}{|l|c|c|c|c|}
\hline \multicolumn{1}{|c|}{ Motivos de interés en la zona } & $\begin{array}{c}\text { Directivos y } \\
\text { profesionales }\end{array}$ & Intermedios & $\begin{array}{c}\text { Trabajadores } \\
\text { manuales }\end{array}$ & Total \\
\hline Estar cerca de la familia & 36 & 47 & 37 & 40 \\
\hline Estar cerca de los amigos & 15 & 19 & 14 & 16 \\
\hline Calidad residencial & 16 & 10 & 7 & 10 \\
\hline Buenas comunicaciones & 22 & 15 & 14 & 17 \\
\hline Bien comunicado con trabajo & 29 & 29 & 27 & 27 \\
\hline Precio & 10 & 14 & 26 & 17 \\
\hline Hay mucha oferta & 3 & 3 & 4 & 4 \\
\hline $\begin{array}{l}\text { Hay más viviendas del tipo } \\
\text { buscado }\end{array}$ & 4 & 5 & 9 & 7 \\
\hline Equipamiento para los niños & 1 & 4 & 3 & 3 \\
\hline Oferta comercial de servicios & 6 & 7 & 2 & 4 \\
\hline Céntrico, bien situado & 10 & 5 & 4 & 6 \\
\hline Buen ambiente & 4 & 3 & 3 & 3 \\
\hline Seguridad & 2 & 2 & 1 & 2 \\
\hline Le gusta / resulta agradable & 11 & 8 & 12 & 12 \\
\hline No quiere cambiar & 6 & 8 & 9 & 8 \\
\hline Está acostumbrado & 12 & 21 & 11 & 14 \\
\hline
\end{tabular}

Fuente: Encuesta de Demanda, 2003. 
Parece lógico que en torno al $40 \%$ de los hogares jóvenes del municipio de Madrid sean del grupo de mayor nivel socioeconómico, y que otro 30\% sean trabajadores manuales que recurran en el centro a las viviendas más accesibles, bien sea a través del mercado de alquiler o de un mercado de vivienda en peor estado y más barato.

Parece claro, también, que el centro sea una opción más frecuente entre los más jóvenes ya que en su mayor parte no tienen proyectos familiares estables y que a medida que avanza la edad los jóvenes vivan en mayor medida en pareja y aparezcan incluso los hijos y tiendan a localizarse en las zonas metropolitanas. Dentro de las zonas metropolitanas, la estructura de precios de la vivienda los lleva a localizarse en mayor medida en la zona sureste que en el caso de los adultos.

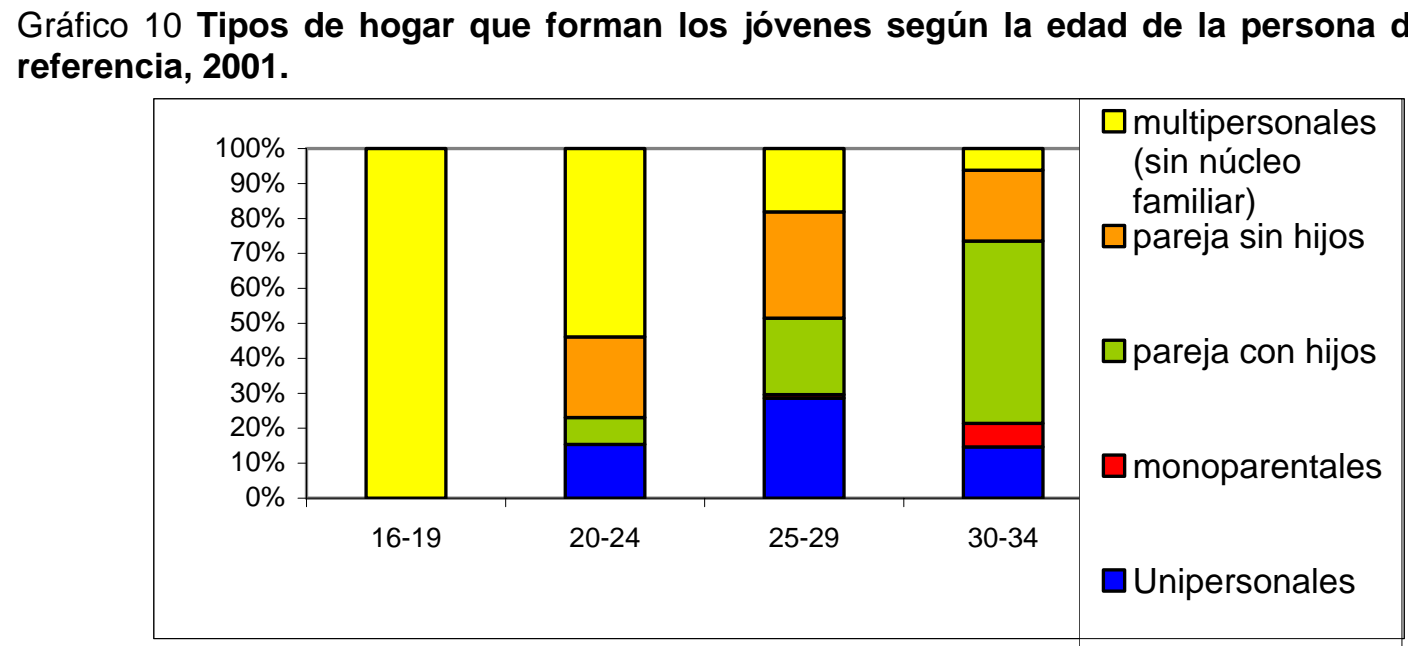

Fuente: Censo de Población y Vivienda.

En definitiva, los jóvenes que se emancipan a edades tempranas tienden a establecerse en hogares unipersonales, "compartiendo piso", y en menor medida a través de la pareja. En estos casos es más frecuente que busquen viviendas en alquiler y dentro del municipio de Madrid que aquellos hogares de mayor edad.

Los jóvenes emancipados en torno a los treinta años, por el contrario, tienden a vivir en núcleos familiares, en viviendas en propiedad y tienden a establecerse en las zonas metropolitanas, especialmente el Sur y el Este, condicionados por la estructura de precios que les empuja hacia zonas más alejadas del centro, lo que explica la mayor presencia de hogares jóvenes en la segunda corona metropolitana. 


\section{Conclusiones}

El mercado inmobiliario está dificultando los procesos de emancipación y el acceso a la vivienda de los hogares jóvenes debido a que no tienen una vivienda previa que capitalizar. Pero este mayor esfuerzo de acceso no explica por si sólo la emancipación tardía ya que otros elementos, como puede ser la creciente preferencia por la compra, pueden estar reteniendo a los jóvenes en sus hogares de origen como una estrategia de acceso. Tener los recursos suficientes parece ser un requisito imprescindible pero no suficiente para dar el paso de establecerse de manera independiente.

Conviene señalar que aunque no sea el único motivo que explique el retraso de la emancipación no podemos ignorar el hecho de que los jóvenes deben hacer un esfuerzo mucho mayor para acceder a un bien básico, fundamental para continuar con su ciclo de vida. Por tanto, los jóvenes disponen de un porcentaje cada vez menor para otros bienes y servicios con los problemas que esto conlleva.

Por otro lado, para una mejor comprensión de las implicaciones que el mercado tiene sobre estos procesos debemos avanzar en el análisis de la relación entre su estructura y las diferentes trayectorias residenciales de los jóvenes. 


\section{REFERENCIAS BIBLIOGRÁFICAS}

Bendit, René y Stokes, Dermot (2004) "Jóvenes en Situación de desventaja Social: políticas de transición entre la construcción social y las necesidades de una juventud vulnerable" en Estudios de Juventud, n65, Madrid, INJUVE, pp. 115-131.

Cabré, Ana (1993) "Volverán tórtolos y cigüeñas" en Garrido, L. y Gil Calvo, E. Estrategias familiares. Madrid, Alianza Editorial.

Casar, Joaquim; García, Maribel; Merino, Rafael y Quesada, Miguel (2006) "Aportaciones teóricas y metodológicas a la sociología de la juventud desde la perspectiva de la transición" en Papers. Revista de socilogia, nº79, Barcelona, Universitat Autònoma de Barcelona, pp.21-48.

(2006b) "Changes in forms of transition in contexts of informational capitalism" en Papers. Revista de sociologia, $\mathrm{n}^{\circ}$ 79, Barcelona, Universitat Autònoma de Barcelona, pp.195224.

Das Dores Guerrero, María y Abrantes, Pedro (2005) "Transiciones a la vida adulta en la era de la globalización. Recorridos de incertidumbre" Recerca. Revista de pensament $i$ anàlisi, $n^{\circ} 5$, Castellón, Universitat Jaime I.

De la Garza Toledo, Enrique (2001) "Subjetividad, Cultura y Estructura", Revista Iztapalapa, no 50, México, pp. 83-104.

Du Bois-Reymond, Manuela y López Blasco, Andreu (2004) "Transiciones tipo yo-yo y trayectorias fallidas: hacia las políticas integradas de transición para los jóvenes europeos" en Estudios de Juventud, n65, Madrid, INJUVE, pp. 11-29.

Elster, Jon (1988) Uvas amargas. La subversión de la racionalidad, Barcelona, Península

Furlong, Andy; Cartmel, Fred y Biggart, Andy (2006) "Choice biographies and transitional linearity: Re-conceptualising modern youth transitions" en Papers. Revista de sociologia, n79, Barcelona, Universitat Autònoma de Barcelona, pp. 225-240.

Garrido Medina, Luis y Gil Calvo, Enrique (1993) "El concepto de estrategias familiares" en Garrido, L. y Gil Calvo, E. Estrategias Familiares, Madrid, Alianza Universidad, pp.13-34.

Gaviria Sabbah, Sandra (2002) "Retener la juventud o invitarla a abandonar la casa familiar" Estudios de Juventud, n58, Madrid, INJUVE, pp. 1-6.

Gil Calvo, Enrique (2002) "Emancipación tardía y estrategia familiar" Estudios de Juventud, no 58, Madrid, INJUVE, pp.1-9.

Inglehart, Ronald (1992) El cambio cultural en las sociedades industriales avanzadas, Madrid, CIS.

Jones, Gill (1995) Leaving home. Open University Press. Buckingham. United Kingdom.

Jurado Guerrero, Teresa (2003) "La vivienda como determinante de la formación familiar en España desde una perspectiva comparada" Revista de Investigaciones Sociológicas, $n^{\circ} 103$, Madrid, pp. 113-157. 
López Blasco, Andreu (2004) "Familia y transiciones: individualización y pluralización de formas de vida" en Informe de Juventud en España 2004, Madrid, INJUVE.

Miret Gamundi, Pau (1997) "Pasado y presente de las pautas de emancipación juvenil" en Vergés Escuín, R. La edad de emancipación de los jóvenes, Barcelona, CCCB.

Módenes Cabrerizo, Juan Antonio (1998) Flujos espaciales e itinerarios biográficos: la movilidad residencial en el área de Barcelona, Tesis doctoral, Universitat Autònoma de Barcelona, 1998, <http://www.tdcat.cesca.es/TDCat-0531101-120649/index.html>

Tezanos, Jose Felix. (2001) La sociedad dividida. Estructuras de clases y desigualdades en las sociedades tecnológicas. Madrid, Biblioteca Nueva.

Zárraga, Jose Luis (1985) Informe Juventud en España. La inserción de los jóvenes en la sociedad, Madrid, Instituto de la Juventud. 